We submit that the contribution of myocardial fibrosis to risk reclassification is therefore more precisely gauged from the individual components of the NRI (ie, the net proportions of patients correctly reclassified among those with and without the outcome). ${ }^{6}$ This information is summarized in our reclassification tables and in the Comment section of the article.

Ankur Gulati, MD

Tevfik F. Ismail, MD

Sanjay K. Prasad, MD

Author Affiliations: Royal Brompton Hospital, London, England (s.prasad@rbht .nhs.uk)

Conflict of Interest Disclosures: The authors have completed and submitted the ICMJE Form for Disclosure of Potential Conflicts of Interest. Dr Gulati reported receiving grants from CORDA, Rosetrees Trust, and the National Institute for Health Research. Dr Ismail reported receiving grant support from the British Heart Foundation. Dr Prasad reported receiving grant support from the British Heart Foundation, CORDA, Rosetrees Trust, and the National Institute for Health Research and receiving speaking fees from Bayer Schering.

1. McMurray JJ, Adamopoulos S, Anker SD, et al; ESC Committee for Practice Guidelines. ESC guidelines for the diagnosis and treatment of acute and chronic heart failure 2012: the Task Force for the Diagnosis and Treatment of Acute and Chronic Heart Failure 2012 of the European Society of Cardiology: developed in collaboration with the Heart Failure Association (HFA) of the ESC. Eur Heart J. 2012 33(14):1787-1847.

2. Levy WC, Linker DT. Prediction of mortality in patients with heart failure and systolic dysfunction. Curr Cardiol Rep. 2008;10(3):198-205.

3. Ismail TF, Prasad SK, Pennell DJ. Prognostic importance of late gadolinium enhancement cardiovascular magnetic resonance in cardiomyopathy. Heart. 2012; 98(6):438-442

4. Pfisterer $M$, Buser $P$, Rickli $H$, et al; TIME-CHF Investigators. BNP-guided vs symptom-guided heart failure therapy: the Trial of Intensified vs Standard Medical Therapy in Elderly Patients With Congestive Heart Failure (TIME-CHF) randomized trial. JAMA. 2009;301(4):383-392.

5. Hunt SA, Abraham WT, Chin MH, et al. 2009 focused update incorporated into the ACC/AHA 2005 guidelines for the diagnosis and management of heart failure in adults: a report of the American College of Cardiology Foundation/ American Heart Association Task Force on Practice Guidelines: developed in collaboration with the International Society for Heart and Lung Transplantation. Circulation. 2009:119(14):e391-e479.

6. Pepe MS. Problems with risk reclassification methods for evaluating prediction models. Am J Epidemiol. 2011;173(11):1327-1335.

\section{Overuse of Emergency Departments}

To the Editor: As in the United States, the overuse and misuse of emergency departments (EDs) is a prominent policy issue in England. The annual number of hospital ED visits in England increased from 15.4 million in 2007-2008 to 18.4 million in 2011-2012. ${ }^{1}$ The patient was given advice only or did not receive treatment in approximately $48 \%$ of these ED visits, and no investigations were performed in approximately $40 \%$ of ED visits. ${ }^{1}$

Dr Adams ${ }^{2}$ commented on the implications of low acuity or nonurgent ED visits for health care costs but did not discuss other reasons for considering these visits as important to policy. A consultation in an ED could be less suitable than one provided by a primary care physician if a patient's condition would benefit from continuity of care or a discussion of nonclinical factors.

Furthermore, nonurgent ED visits use resources, including time, for which other patients may have a greater clinical need. One response to the overuse and misuse of EDs in England has been to design services in which nonemer- gency patients are unable to access the ED unless directed by a primary care physician working in a center co-located with the ED. ${ }^{3}$ The cost-effectiveness of this form of service delivery is yet to be determined, but it is presumed to prevent conditions that are treatable in primary care from reaching the ED, thereby reducing ED workload.

The role of primary care physicians in initiating and directing hospital care also has received attention in the United States. ${ }^{4}$ Adams ${ }^{2}$ suggested that reform policies should focus on "... patients who generate the highest costs, such as those who use the ED most frequently...." This implies that interventions should focus on patients at greatest risk of visiting an ED.

However, Rose ${ }^{5}$ highlighted the limitations of the highrisk strategy several decades ago; a large number of patients at low risk of visiting an ED may make a greater number of ED visits than a small number at high risk. Consequently, a population strategy that attempts to lower the mean level of risk for the whole population could have a larger effect on reducing ED visits.

The solutions offered by Adams, ${ }^{2}$ such as improving access to primary care physicians and the integration of care between EDs and other health services, lend themselves well to the population approach and may reduce both nonurgent and urgent ED visits.

Thomas E. Cowling, MPH

Azeem Majeed, MD

Author Affiliations: Department of Primary Care and Public Health, Imperial College London, London, England (t.cowling@imperial.ac.uk).

Conflict of Interest Disclosures: The authors have completed and submitted the ICMJE Form for Disclosure of Potential Conflicts of Interest and none were reported.

1. Health and Social Care Information Centre. Accident and emergency attendances in England. http://www.hscic.gov.uk/searchcatalogue?q=title \%3A $\% 22$ accident+and+emergency+attendances $\% 22 \&$ area $=\&$ size $=10 \&$ sort=Relevance \&topics=0\%2FHospital+care. Accessed May 10, 2013.

2. Adams JG. Emergency department overuse: perceptions and solutions. JAMA. 2013;309(11):1173-1174.

3. Healthcare for London. A service delivery model for urgent care centres: commissioning advice for PCTs. http://www.londonhp.nhs.uk/wp-content/uploads /2011/03/Urgent-care-centres-delivery-model.pdf. Accessed May 10, 2013.

4. Schuur JD, Venkatesh AK. The growing role of emergency departments in hospital admissions. N Engl J Med. 2012;367(5):391-393.

5. Rose G. Sick individuals and sick populations. Int J Epidemiol. 1985;14(1): 32-38.

In Reply: The suggestion of Mr Cowling and Dr Majeed to ensure the availability of primary care when and where the patients need it should be encouraged. As new models to increase the accessibility of primary care services are developed and evaluated, policy makers must be careful to avoid a priori assumptions that EDs are overused and misused because of poor decisions on the part of patients.

The reasons people visit the ED for care are often rational and understandable, driven by a perceived need for immediacy of treatment and lack of an accessible alternative. ${ }^{1}$ Many conditions treatable in primary care that are cared for inside EDs in the United States may reflect problems with 
alternative access to rather than inappropriate actions by the patient. $^{2}$

More than $50 \%$ of all acute care visits by the uninsured are to emergency physicians, who comprise less than $5 \%$ of the total number of physicians in the United States. ${ }^{3}$ In addition, for the general population, we must consider the possibility that EDs are not, in fact, significantly overused at all.

Evidence would suggest that people are not increasingly reliant on EDs to meet ambulatory care needs. Between 1999 and 2009, total ambulatory visits increased from 841 million to 1.13 billion, or 3720 visits per 1000 people annually. ${ }^{4}$ During this same period, the number of ED visits increased from approximately 108 million to 124 million, which is a slower rate of growth.,

In other words, total ambulatory visits increased more quickly than ED visits, implying that patients are finding accessible ambulatory care, at least if they have insurance. Visits to the ED currently make up a lower proportion of total ambulatory visits than the prior decade. The growth of ED visits might not signal a problem at all, but rather that our health care system is undergoing fundamental change.

This change includes a shift away from inpatient use and toward outpatient care, especially for the increasing numbers of patients with complex illnesses. ${ }^{4}$ During the same period, there has been a decline in hospitalizations, which would not have been achievable without highly effective ambulatory services, backed up by 24-hour, relatively unconstrained access to high-intensity, high-quality emergency care.

As policy makers look to optimize models for health care delivery, it should not be presumed that that ED use rates, in isolation, are somehow an indicator of quality or cost-effectiveness. Immediate ED access is an important part of the portfolio of health care services provided to a population.

The proposal by Cowling and Majeed to focus on population health is quite wise. Reducing the cost of health care is most effectively achieved when the need for health care is reduced. In doing so, policy makers should focus less on ED use alone and instead focus on whether patients have accessible, cost-effective medical services that will achieve desired health outcomes. As coordinated solutions to achieve this end are implemented, the absolute number of ED visits may not decrease, although their proportion in relation to overall ambulatory visits may indeed decline further.

James G. Adams, MD

Author Affiliation: Department of Emergency Medicine, Northwestern University Feinberg School of Medicine, Chicago, Illinois (jadams@nmh.org).

1. Gindi RM, Cohen RA, Kirzinger WK. Emergency room use among adults aged 18-64: early release of estimates from the National Health Interview Survey, JanuaryJune 2011. http://www.cdc.gov/nchs/nhis/releases.htm. Accessed April 30, 2013 2. Kellermann AL, Weinick RM. Emergency departments, Medicaid costs, and ac cess to primary care-understanding the link. N Engl J Med. 2012;366(23): 2141-2143.

3. Pitts SR, Carrier ER, Rich EC, Kellermann AL. Where Americans get acute care increasingly, it's not at their doctor's office. Health Aff (Millwood). 2010;29 (9):1620-1629

2550 JAMA, June 26, 2013-Vol 309, No. 24
4. Barnett $M L$, Song $Z$, Landon BE. Trends in physician referrals in the United States, 1999-2009. Arch Intern Med. 2012:172(2):163-170.

5. National Center for Health Statistics. NAMCS/NHAMCS main site. http://www .cdc.gov/nchs/ahcd.htm. Accessed April 30, 2013.

\section{RESEARCH LETTER}

\section{Prevalence and Correlates of Traumatic Brain Injuries Among Adolescents}

To the Editor: Traumatic brain injury (TBI) among adolescents has been identified as an important health priority. ${ }^{1,2}$ However, studies of TBI among adolescents in large representative samples are lacking. ${ }^{1,2}$ This information is important to the planning and evaluation of injury prevention efforts, particularly because even minor TBI may have important adverse consequences. ${ }^{2}$

We describe the prevalence of TBI, mechanisms of injury, and adverse correlates in a large representative sample of adolescents living in Ontario, Canada.

Methods. Data were derived from the Centre for Addiction and Mental Health's 2011 Ontario student drug use and health survey, consisting of anonymous, self-administered questionnaires completed in classrooms (62\% response rate) by students grades 7-12 (age range: 11-20 years). A complete description of the study, including design and discussion of the validity of self-reports, potential nonresponse bias, and limitations, is available. ${ }^{3}$

Traumatic brain injury was defined as an acquired head injury in which the student was unconscious for at least 5 minutes or hospitalized overnight. ${ }^{4}$ Students were asked if they ever had such injury in the past 12 months or in their lifetime (excluding the past 12 months). Students reporting TBI in the past 12 months were asked about the source of the injury. Questions about consumption of alcohol and cannabis use during the last 12 months and usual grades received were also included.

All participants provided signed and parental consent. Research ethics board approval was provided by the Centre for Addiction and Mental Health, York University, and public and Catholic school boards throughout Ontario. The surveys were administered during a class period by field staff. Analyses were based on a complex sample design with 15 strata (region $\times$ school level), 181 primary sampling units (schools), and 8915 students.

All analyses used Taylor series linearization available in the complex sample module in SPSS version 20.0 (SPSS Inc). Multinomial logistic regression was performed fitting the 5 factors of sex, grade, past-year alcohol use, past-year cannabis use, and school grades against a 2 -tailed $P<.05$.

Results. The mean (SD) age of participants was 15.1 (1.82) years. The estimated lifetime prevalence of TBI was $20.2 \%$ (95\% CI, 18.1\%-22.4\%); 5.6\% (95\% CI, 4.2\%-7.5\%) reported at least $1 \mathrm{TBI}$ in the past 12 months (4.3\% of girls and $6.9 \%$ of boys) and $14.6 \%$ (95\% CI, 13.4\%-15.9\%) re-

C2013 American Medical Association. All rights reserved. 